\title{
Generation and detection of pure valley current by electrically induced Berry curvature in bilayer graphene
}

\author{
Y. Shimazaki ${ }^{1}$, M. Yamamoto ${ }^{1,2 \star}$, I. V. Borzenets ${ }^{1}$, K. Watanabe ${ }^{3}$, T. Taniguchi ${ }^{3}$ and S. Tarucha ${ }^{1,4 \star}$
}

The field of 'Valleytronics' has recently been attracting growing interest as a promising concept for the next generation electronics, because non-dissipative pure valley currents with no accompanying net charge flow can be manipulated for computational use, akin to pure spin currents'. Valley is a quantum number defined in an electronic system whose energy bands contain energetically degenerate but non-equivalent local minima (conduction band) or maxima (valence band) due to a certain crystal structure. Specifically, spatial inversion symmetry broken two-dimensional honeycomb lattice systems exhibiting Berry curvature is a subset of possible systems that enable optical ${ }^{2-5}$, magnetic ${ }^{6-9}$ and electrical control of the valley degree of freedom ${ }^{10-12}$. Here we use dual-gated bilayer graphene to electrically induce and control broken inversion symmetry (or Berry curvature) as well as the carrier density for generating and detecting the pure valley current. In the insulating regime, at zero-magnetic field, we observe a large nonlocal resistance that scales cubically with the local resistivity, which is evidence of pure valley current.

Charge and spin are both well-defined quantum numbers in solids. Spintronics is a technology that uses the spin degree of freedom. The application range of spintronics has been largely expanded by the development of electrical techniques for generating and detecting the spin current ${ }^{1}$. The valley degree of freedom in solid crystals can be handled by controlling the occupation of the non-equivalent structures in the band, providing the novel concept of so-called valleytronics.

Among various material candidates for valleytronics, twodimensional (2D) honeycomb lattice systems with broken spatial inversion symmetry, such as gapped graphene and transition metal dichalcogenide (TMDC), are predicted to be the most useful. These systems have two valleys, called $\mathrm{K}$ and $\mathrm{K}^{\prime}$. Optical ${ }^{2-5}$, magnetic ${ }^{6-9}$ and electrical ${ }^{10-12}$ control of the valley has been demonstrated. In particular, Berry curvature, which emerges in these honeycomb lattice systems with broken spatial inversion symmetry, enables electrical control of the valley degree of freedom.

Berry curvature acts as an out-of-plane pseudo-magnetic field in momentum space and has opposite sign between the two valleys. Therefore, a transverse pure valley current is generated by means of the anomalous velocity, in analogy to a transverse electronic current being generated by means of the Lorentz force due to a magnetic field in real space ${ }^{13,14}$ (see Fig. 1d). This phenomenon is called the valley Hall effect ${ }^{10}$ and can be used to generate a valley current. The inverse valley Hall effect, which converts the valley current into a transverse electric field, allows the detection of the pure valley current.

The valley Hall effect was first reported for photo-generated electrons in monolayer $\mathrm{MoS}_{2}$ (ref. 11). However, the small intervalley scattering length in this material prevents the detection of the pure valley current, which does not accompany the electronic current. Compared to TMDC, graphene has a much larger inter-valley scattering length owing to its higher crystal quality. Monolayer graphene on h-BN has more recently been used to generate and detect the pure valley current, where the crystal direction of the graphene was aligned to that of the h-BN such that the superlattice potential imposed by the h-BN structurally breaks the spatial inversion symmetry ${ }^{12}$. The valley Hall effect was analysed in detail with the carrier density as a parameter using metallic samples whose resistivity decreases as the temperature is lowered, but leaving unaddressed the insulating regime, which is more appropriate for investigating the pure valley current.

In this work we employed bilayer graphene (BLG) to generate and detect the valley current. We used a perpendicular electric field to break the spatial inversion symmetry and induce Berry curvature as well as a bandgap (see Fig. 1a). The dual-gated structure seen in Fig. $1 \mathrm{~b}$ allows electrical and independent control of the perpendicular electric field and the carrier density ${ }^{15-19}$. This is in contrast to the monolayer graphene samples in ref. 12, where the monolayer graphene has to be structurally aligned with h-BN through a process of mechanical transfer. BLG valley Hall devices therefore show greater promise in terms of tunability of the valley current and applications to electronic devices. Indeed we show that independent control of the Fermi level and the bandgap enables us to prove the existence of the valley Hall effect in the insulating regime where the local resistivity increases with decreasing temperature. The significant advantage of the insulating system is that conversion from the electric field to the valley current is less dissipative than that in the metallic regime, as a much smaller current is injected. Such a regime has not been accessible with conventional spin or valley Hall systems.

In bilayer graphene with broken spatial inversion symmetry, the Berry curvature $\Omega$ and intrinsic valley Hall conductivity $\sigma_{x y}^{\mathrm{VH}}$ are calculated as a function of the Fermi energy $E_{\mathrm{F}}$ (refs 10,20,21):

$$
\Omega\left(E_{\mathrm{F}}\right)=\tau_{z} \frac{\hbar^{2}}{m} \frac{\Delta \sqrt{E_{\mathrm{F}}^{2}-\Delta^{2}}}{E_{\mathrm{F}}^{3}}
$$

\footnotetext{
${ }^{1}$ Department of Applied Physics, University of Tokyo, Bunkyo-ku, Tokyo 113-8656, Japan. ${ }^{2}$ PRESTO, JST, Kawaguchi-shi, Saitama 332-0012, Japan. ${ }^{3}$ National Institute for Materials Science, Tsukuba-shi, Ibaraki 305-0044, Japan. ${ }^{4}$ Center for Emergent Matter Science (CEMS), RIKEN, Wako-shi, Saitama 351-0198, Japan. ^e-mail: yamamoto@ap.t.u-tokyo.ac.jp; tarucha@ap.t.u-tokyo.ac.jp
} 

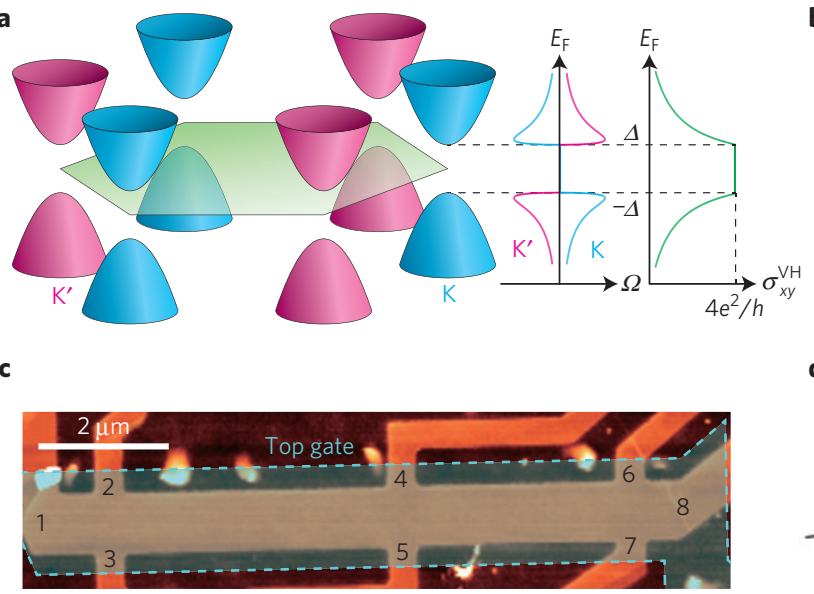

b

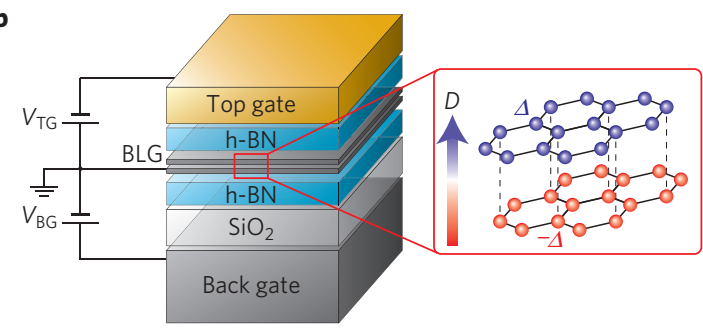

d

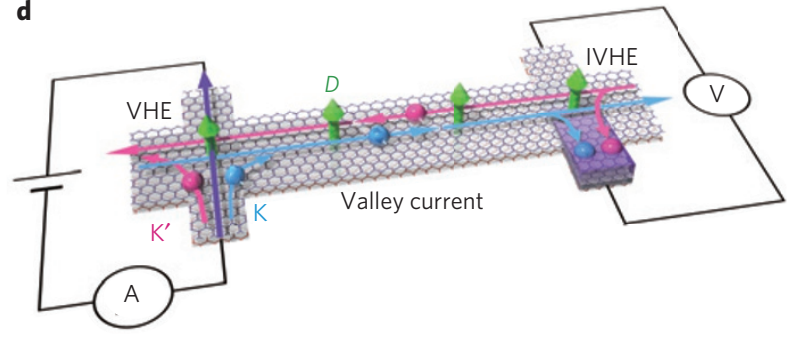

Figure 1 | Scheme for detection of the nonlocal resistance due to valley current flow in BLG. a, Band structure, Berry curvature and valley Hall conductivity of BLG. A band gap $2 \Delta$ and Berry curvature $\Omega$ emerge as a result of broken spatial inversion symmetry. The valley Hall conductivity $\sigma_{x y}^{\mathrm{VH}}$, which is calculated by integrating the Berry curvature, is constant in the bandgap. $\mathbf{b}$, Schematic of the dual-gated BLG device. The top gate is a gold film, and the

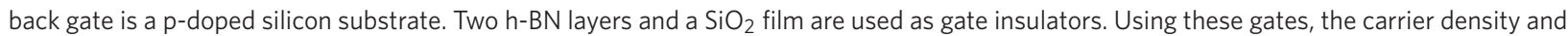
perpendicular electric field (displacement field) are independently varied. Expanded image: Lattice structure of AB-stacked BLG. In the presence of a displacement field, an energy difference between the top and bottom layer emerges. Therefore spatial inversion symmetry is broken in this system, and both Berry curvature and a bandgap emerge. c, AFM image of the BLG device without the top h-BN. The light blue region indicates the area of the top gate. The BLG has a mobility of $\sim 15,000 \mathrm{~cm}^{2} \mathrm{~V}^{-1} \mathrm{~s}^{-1}$ at both 1.5 and $70 \mathrm{~K}$. d, Schematic of the nonlocal resistance measurement and the nonlocal transport mediated by a pure valley current. The electric field driving the charge current in the left-hand circuit generates a pure valley current in the transverse direction by means of the valley Hall effect (VHE). This valley current is converted into an electric field or nonlocal voltage in the right-hand circuit by means of the inverse valley Hall effect (IVHE) to generate the nonlocal resistance.

and

$$
\sigma_{x y}^{\mathrm{VH}}\left(E_{\mathrm{F}}\right)= \begin{cases}\frac{4 e^{2}}{h} \frac{\Delta}{\left|E_{\mathrm{F}}\right|} & \left(\left|E_{\mathrm{F}}\right| \geq \Delta\right) \\ \frac{4 e^{2}}{h} & \left(\left|E_{\mathrm{F}}\right|<\Delta\right)\end{cases}
$$

where $\tau_{z}$ is the valley index $\left(\tau_{z}=-1\right.$ for $\mathrm{K}$ and +1 for $\left.\mathrm{K}^{\prime}\right), m$ is the effective mass in BLG without broken spatial inversion symmetry, $e$ is the elementary charge, $h$ is the Planck constant, and $\hbar=h / 2 \pi$. The Berry curvature $\Omega$ is defined only for $\left|E_{\mathrm{F}}\right| \geq \Delta$ (half the bandgap, see Fig. 1a). $\sigma_{x y}^{\mathrm{VH}}$ saturates at the maximum value $4 e^{2} / h$ when the Fermi level lies in the gap, because all occupied states in the valence band contribute to the valley Hall effect. Away from the gap-for example, when the Fermi energy lies above the gap-the conduction band, which has the opposite sign of Berry curvature to that of the valence band, contributes to reduce $\sigma_{x y}^{\mathrm{VH}}$.

To detect the pure valley current, the nonlocal resistance $R_{\mathrm{NL}}$ was measured in the same scheme as is widely used in the spintronics field to detect pure spin current ${ }^{22-26}$. We observed a value of $R_{\mathrm{NL}}$ at the charge neutrality point in the presence of a perpendicular electric field that was three orders of magnitude larger than the $R_{\mathrm{NL}}$ due to the Ohmic contribution (explained later). We also found a cubic scaling relation between $R_{\mathrm{NL}}$ and resistivity $\rho\left(=1 / \sigma_{x x}\right)$, which is expected to appear when $\sigma_{x x}$ is much larger than $\sigma_{x y}^{\mathrm{VH}}$ in the intrinsic valley Hall effect. This cubic scaling was reproduced in multiple devices. From these findings we conclude that the origin of the observed large nonlocal resistance is the transport mediated by pure bulk valley current in a gapped state with electrically induced Berry curvature.

Figure $1 \mathrm{~b}, \mathrm{c}$ shows the schematic of the dual-gated BLG device and an AFM image of the device, respectively. BLG is encapsulated between two h-BN layers ${ }^{27}$ (see Methods) and gated through the $\mathrm{h}-\mathrm{BN}$ layer from the top and from the bottom. The local and nonlocal resistance $R_{\mathrm{L}}$ and $R_{\mathrm{NL}}$ were derived from measurements of the four-terminal resistance $R_{i j, k l}$, which is defined by the voltage between terminals $i$ and $j$ divided by the charge current injected between terminals $k$ and $l$ (see Fig. 1c,d). Unless mentioned, $R_{\mathrm{L}}$ and $R_{\mathrm{NL}}$ denote $R_{57,38}$ and $R_{45,67}$, respectively. The measurement was performed at $70 \mathrm{~K}$ using a low-frequency (around $1 \mathrm{~Hz}$ ) lockin technique, unless mentioned (see Methods and Supplementary Section I for details of the measurement).

Figure 2a,b shows the gate voltage dependence of $R_{\mathrm{L}}$ and $R_{\mathrm{NL}}$, respectively. At the charge neutrality point $(\mathrm{CNP}), R_{\mathrm{L}}$ increases with the displacement field $(D)$ (see Fig. 2a), reflecting the bandgap opening due to inversion symmetry breaking ${ }^{15-19,28}$. We found that $R_{\mathrm{NL}}$ also increases with $D$ around the CNP.

In analogy with the spin Hall effect ${ }^{23}$ (Supplementary Section IX), $R_{\mathrm{NL}}$ arising from the valley Hall and inverse valley Hall effects is given by

$$
R_{\mathrm{NL}}=\frac{1}{2}\left(\frac{\sigma_{x y}^{\mathrm{VH}}}{\sigma_{x x}}\right)^{2} \frac{W}{\sigma_{x x} l_{\mathrm{v}}} \exp \left(-\frac{L}{l_{\mathrm{v}}}\right)
$$

where $\sigma_{x y}^{\mathrm{VH}}$ and $l_{\mathrm{v}}$ are the valley Hall conductivity and the inter-valley scattering length, respectively. $W$ and $L$ are the width and length of the Hall bar channel. Local conductivity $\sigma_{x x}$ is minimized at the CNP and with increasing $D$, thus enhancing $R_{\mathrm{NL}}$ (equation (3)). For a given $D, R_{\mathrm{NL}}$ is further maximized around the CNP owing to the maximal valley Hall conductivity $\sigma_{x y}^{\mathrm{VH}}$ (equation (2)). We confirmed that $R_{\mathrm{NL}}$ is unchanged when swapping the measurement terminals-that is, $R_{45,67} \sim R_{67,45}$ (Supplementary Section II). We also consider a contribution of trivial Ohmic resistance which is due to classical diffusive charge transport to the measured nonlocal resistance. The Ohmic contribution can be calculated using the van der Pauw formula $R_{\mathrm{NL}}=\rho / \pi \exp (-\pi(L / W)$ ) (refs 12,24-26), where we define the resistivity $\rho=R_{\mathrm{L}}(W / L)$, and is compared with the measured nonlocal resistance in Fig. $2 \mathrm{c}$. The measured $R_{\mathrm{NL}}$ is three 


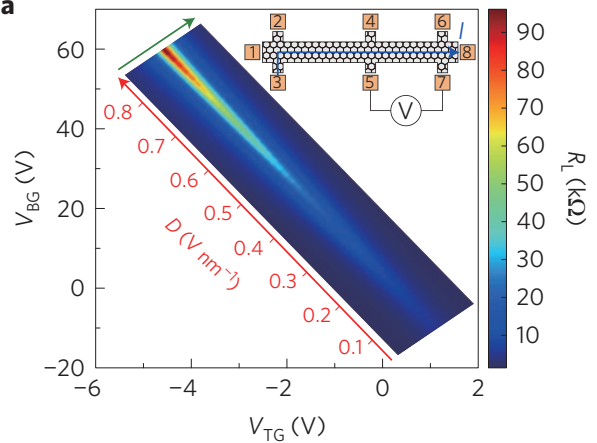

b

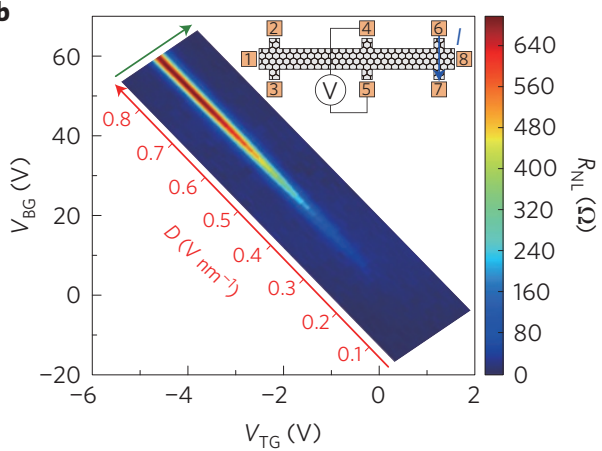

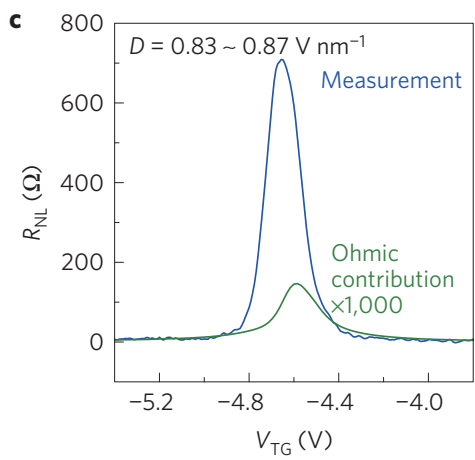

Figure 2 | Measured local and nonlocal resistances $R_{\mathrm{L}}$ and $R_{\mathrm{NL}} \cdot \mathbf{a}, \mathbf{b}$, Gate voltage dependence of $R_{\mathrm{L}}$ and $R_{\mathrm{NL}}$, respectively. The displacement fields from the back and top gate are defined as $D_{\mathrm{BG}}=\varepsilon_{\mathrm{BG}}\left(V_{\mathrm{BG}}-V_{\mathrm{BG}}^{0}\right) / d_{\mathrm{BG}}$ and $D_{\mathrm{TG}}=-\varepsilon_{\mathrm{TG}}\left(V_{\mathrm{TG}}-V_{\mathrm{TG}}^{0}\right) / d_{\mathrm{TG}}$, respectively, where $\varepsilon_{\mathrm{BG}}\left(\varepsilon_{\mathrm{TG}}\right)$ and $d_{\mathrm{BG}}\left(d_{\mathrm{TG}}\right)$ are the relative dielectric constant and thickness of the back (top) gate, respectively and $V_{\mathrm{BG}}^{0}\left(V_{\mathrm{TG}}^{0}\right)$ is the offset of the back (top) gate voltage under the top gated region due to environmental doping. The displacement field $D$ is defined by the average of $D_{B G}$ and $D_{T G}$. The red axis shows the scale of $D$. Inset: Schematic of the measurement configuration. The blue arrow shows the direction of charge flow. c, Comparison of the measured $R_{\mathrm{NL}}$ (blue) with a calculation of the Ohmic contribution (magnified 1,000 times) (green). The $R_{N L}$ curve is extracted from the data along the direction of the green arrow in $\mathbf{b}$ at the highest $D$. The Ohmic contribution curve is calculated using the $R_{\mathrm{L}}$ data along the direction of the green arrow in $\mathbf{a}$ at the highest $D$ (see text).
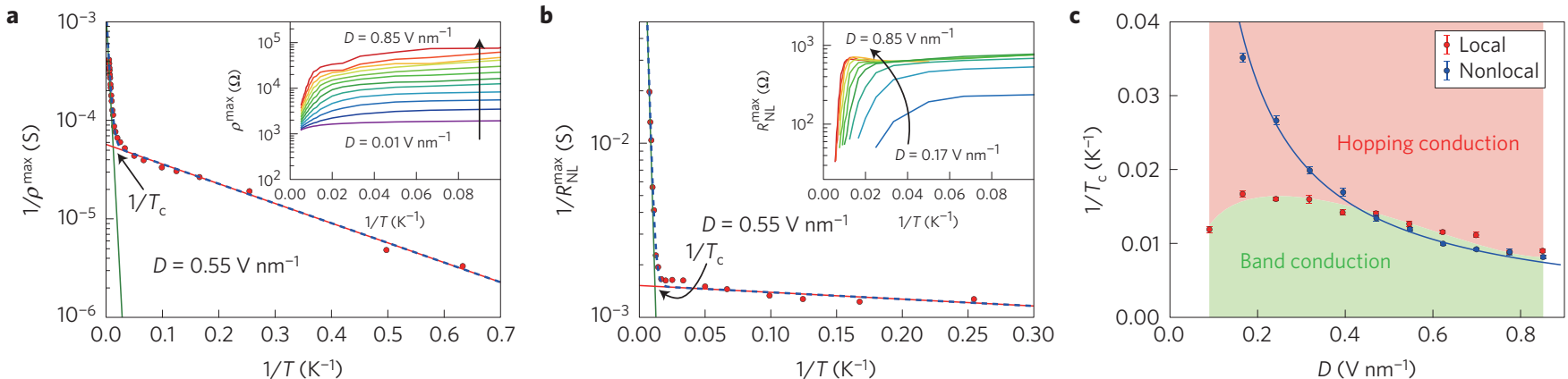

Figure 3 | Temperature dependence of $\rho^{\max }$ and $R_{\mathrm{NL}}^{\max }, \mathbf{a}, \mathbf{b}$, Typical data fitting using a double exponential function for $1 / \rho^{\max }(\mathbf{a})$ and $1 / R_{\mathrm{NL}}^{\max }(\mathbf{b})$. The blue broken curve indicates the fitting curve. The green (red) line indicates the contribution from band (hopping) conduction. The crossover temperature $T_{\mathrm{C}}$ is defined by the crossing of the two lines. Inset: Temperature dependence of maximum $\rho$ (a) and $R_{\mathrm{NL}}$ (b) with respect to the carrier density. $D$ is varied from $0.85 \mathrm{~V} \mathrm{~nm}^{-1}$ (red) to $0.01 \mathrm{~V} \mathrm{~nm}^{-1}$ (purple) in $\mathbf{a}$ and from $0.85 \mathrm{~V} \mathrm{~nm}^{-1}$ (red) to $0.17 \mathrm{~V} \mathrm{~nm}^{-1}$ (blue) in $\mathbf{b}$ with a constant interval. $\mathbf{c}, T_{\mathrm{c}}$ derived from the data fitting as in $\mathbf{a}$ for $1 / \rho^{\max }$ and $\mathbf{b}$ for $1 / R_{\mathrm{NL}}^{\max }$. The error bars are due to the accuracy of the fitting. The light green (red) area is the region of band conduction (hopping conduction). The blue curve shows the fitting result for the nonlocal $1 / T_{\mathrm{C}}$ versus $1 / D$.

orders of magnitude larger than the calculated Ohmic contribution. We therefore exclude the Ohmic contribution as the origin of the observed $R_{\mathrm{NL}}$.

In the gapped BLG, the electron conduction mechanism depends on the temperature $T$. At high $T$ it is dominated by thermal activation across the bandgap, namely band transport, whereas at low $T$ it is dominated by hopping conduction between impurity states ${ }^{15,17-19}$.

The temperature dependence of maximum $\rho$ with respect to carrier density $\left(\rho^{\max }\right)$ was measured for various displacement fields $D$ (Fig. 3a, inset). We plot $1 / \rho^{\max }$ as a function of $1 / T$ for $D=0.55 \mathrm{~V} \mathrm{~nm}^{-1}$ as a typical example in Fig. 3a. The temperature dependence is strong at high $T$ (>79 K), reflecting band conduction, and weak at low $T$, reflecting hopping conduction. The temperature dependence over the whole range is reproduced well by a double exponential function:

$$
\frac{1}{\rho^{\max }}=\frac{1}{\rho_{1}} \exp \left(-\frac{E_{1}^{\mathrm{L}}}{k_{\mathrm{B}} T}\right)+\frac{1}{\rho_{2}} \exp \left(-\frac{E_{2}^{\mathrm{L}}}{k_{\mathrm{B}} T}\right)
$$

where $E_{1}^{\mathrm{L}}\left(E_{2}^{\mathrm{L}}\right)$ and $\rho_{1}\left(\rho_{2}\right)$ are the activation energy and the local resistivity, respectively for the high- $T$ (low- $T$ ) regime. $2 E_{1}^{\mathrm{L}}$ indicates the bandgap size, and is around $80 \mathrm{meV}$ at the highest $D$ (Supplementary Section IV). The crossover temperature $T_{\mathrm{c}}$ between the high- and low-temperature regions is determined by the crossing point of the first and second term of equation (4), as shown in Fig. 3a. The temperature dependence of the maximum nonlocal resistance $R_{\mathrm{NL}}^{\max }$ was also measured (Fig. 3b, inset) and analysed with the following fitting function in the same way as for $\rho^{\max }$, as shown in Fig. 3b:

$$
\frac{1}{R_{\mathrm{NL}}^{\max }}=\frac{1}{R_{1}} \exp \left(-\frac{E_{1}^{\mathrm{NL}}}{k_{\mathrm{B}} T}\right)+\frac{1}{R_{2}} \exp \left(-\frac{E_{2}^{\mathrm{NL}}}{k_{\mathrm{B}} T}\right)
$$

where $E_{1}^{\mathrm{NL}}\left(E_{2}^{\mathrm{NL}}\right)$ is the activation energy and $R_{1}\left(R_{2}\right)$ is a fitted proportionality factor, respectively, for the high- $T$ (low- $T$ ) regime. The temperature dependence is fairly similar to that of $\rho^{\max }$ in Fig. 3a. We also plot the crossover temperature $T_{\mathrm{c}}$ for both $1 / \rho^{\max }$ and $1 / R_{\mathrm{NL}}^{\max }$ as a function of $D$ in Fig. $3 \mathrm{c}$. The $T_{\mathrm{c}}$ for $1 / \rho^{\max }$ divides the $D-T$ plane into the band conduction region (light green) and the hopping conduction region (light red).

The critical temperatures $T_{\mathrm{c}}$ of $1 / \rho^{\max }$ and $1 / R_{\mathrm{NL}}^{\max }$ coincide for $D>0.4 \mathrm{~V} \mathrm{~nm}^{-1}$, indicating that there is correlation of the crossover behaviour between the local and nonlocal transport. However, it deviates for $D<0.4 \mathrm{~V} \mathrm{~nm}^{-1}$ for the following two possible reasons. The first possible reason is underestimation of the $T_{c}$ of the nonlocal transport in the low- $D$ region. The nonlocal voltage becomes very small at high $T$ and low $D$, making precise measurement 


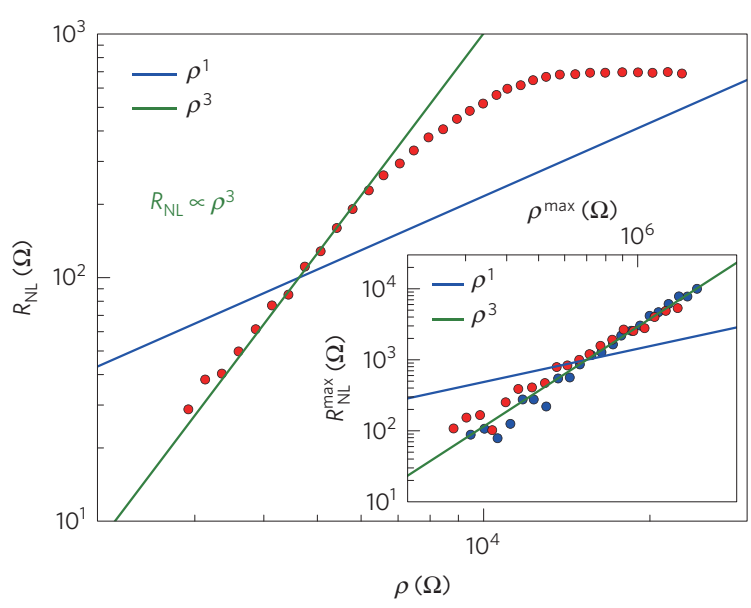

Figure 4 | Scaling relation between $\rho$ and $R_{N L}$ at CNP. Each data point is extracted from Fig. $2 a, b$ for a different $D$ value ranging from 0.22 to $0.85 \mathrm{~V} \mathrm{~nm}^{-1}$ at CNP. Inset: Scaling relation between the maximum $\rho\left(\rho^{\max }\right)$ and $R_{\mathrm{NL}}\left(R_{\mathrm{NL}}^{\max }\right)$ as functions of the carrier density obtained from various $D$ ranging from 0.51 to $0.75 \mathrm{~V} \mathrm{~nm}^{-1}$ (red points) and -0.50 to $-0.76 \mathrm{~V} \mathrm{~nm}^{-1}$ (blue points) in another dual-gated bilayer graphene Hall bar device. The width and length of the Hall bar channel are $1 \mu \mathrm{m}$ and $4.5 \mu \mathrm{m}$, respectively. Measurement was performed at $50 \mathrm{~K}$. At lower temperatures, we also observed deviation from the cubic scaling for high displacement fields.

of $R_{\mathrm{NL}}$ difficult. In this regime, there are fewer measured points available for the fitting, resulting in the underestimation of $T_{c}$. The second possible reason is that the nonlocal transport by the valley current is less affected by charge puddles compared to the local transport, although we do not yet fully understand the reason for this observation.

One noticeable result is that the $T_{c}$ of the nonlocal transport depends almost linearly on $D$ for the entire region in Fig. 3c (see the blue curve; Supplementary Section V). This behaviour may indicate that the $T_{c}$ is affected by the size of bandgap but less affected by the size of potential fluctuations due to charge puddles. Note that all four of the fitting parameters in equations (4) and (5) have a $D$ dependence; therefore, obtaining an analytical relationship between $D$ and $T_{\mathrm{c}}$ is not straightforward.

Another notable result is that the high- $T$ activation energy $E_{1}$ is different between the local and nonlocal transport (Supplementary Sections IV and V, see Supplementary Figs 7 and 8). This already implies there is no linear relation between $R_{\mathrm{NL}}$ and $\rho$ in our device. This observation is in contrast to a previous report on monolayer graphene ${ }^{12}$, where both activation energies were similar.

We now present the scaling relation between $\rho$ and $R_{\mathrm{NL}}$ at the CNP. Figure 4 is a plot of $R_{\mathrm{NL}}$ versus $\rho$ obtained for various displacement fields $D$. The crossover behaviour between the band conduction and the hopping conduction shows up again on this plot. In the band conduction region $(\rho<7 \mathrm{k} \Omega)$ we observe a clear cubic scaling relation (green line), whereas we observe saturation in the hopping conduction region $(\rho>7 \mathrm{k} \Omega)$. Similar cubic and saturating scaling relations are obtained for different physical conditions. The $\rho$ versus $R_{\mathrm{NL}}$ relation obtained for different carrier densities and temperatures at fixed displacement fields are shown in Supplementary Sections VI and VII, respectively. In addition, we observe a similar scaling relation for multiple devices (see Fig. 4 inset for one example).

By assuming a constant inter-valley scattering length and replacing $\sigma_{x x}$ with $\rho^{-1}$ in equation (3), we derive the following scaling relation between $R_{\mathrm{NL}}$ and $\rho$ :

$$
R_{\mathrm{NL}} \propto\left(\sigma_{x y}^{\mathrm{VH}}\right)^{2} \rho^{3}
$$

The cubic scaling between $R_{\mathrm{NL}}$ and $\rho$ holds for the constant valley Hall conductivity which is expected when the Fermi level is in the bandgap or near the CNP (see Fig. 1a) for the intrinsic valley Hall effect, $\sigma_{x y}^{\mathrm{VH}}=4 e^{2} / h$. The observed cubic relation for small $D$ in Fig. 4 is therefore consistent with the theoretical expectation, providing unambiguous evidence of the valley transport. Note that, at finite temperatures, $\sigma_{x y}^{\mathrm{VH}}$ is reduced from $4 e^{2} / h$. However, in the range of displacement fields used here, it stays almost constant with a value close to $4 e^{2} / h$ (see Supplementary Section X).

Using $\sigma_{x y}^{\mathrm{VH}}=4 e^{2} / h$ and substituting $\sigma_{x x}=\rho^{-1}$ and the sample dimensions into equation (3), we obtain $l_{\mathrm{v}}=1.6 \mu \mathrm{m}$. This is comparable to the estimated inter-valley scattering length in previous works ${ }^{12,29}$. By using different sets of four terminals we observed a significantly increasing decay of $R_{\mathrm{NL}}$ with $L$ (Supplementary Section V), probably owing to valley relaxation due to edge scattering, as discussed in a weak localization study ${ }^{29}$.

We here note that equations (3) and (6) are valid only for $\sigma_{x x} \gg \sigma_{x y}^{\mathrm{VH}}$ (Supplementary Section IX). Otherwise we need to solve the conductance matrix and the diffusion equation of the entire Hall bar in a self-consistent way. Indeed, deviation from the cubic scaling in the large- $D$ region observed in Fig. 4 may arise owing to the inapplicability of equations (3) and (6). However, it does not account for the saturation of $R_{\mathrm{NL}}$ for large $\rho$ (Supplementary Section IX). Another possible scenario to account for the saturation of $R_{\mathrm{NL}}$ is the crossover of the conduction mechanism, as discussed in Fig. 3c. In studies of the anomalous Hall effect, the crossover between the metallic and the hopping transport regime has been experimentally studied, and the scaling relation $\sigma_{x y} \propto \sigma_{x x}^{1.6}$ has been reported in a wide range of materials ${ }^{13}$. If we apply this experimental rule for equation (6), we find $R_{\mathrm{NL}}$ to be almost constant with $\rho$. Here, we are again cautious about the validity of equation (6) in this argument, because in the saturation region $\sigma_{x x}<\sigma_{x y}^{\mathrm{VH}}$ for $\sigma_{x y}^{\mathrm{VH}}=4 e^{2} / h$. However, by including extrinsic contributions-for example, the side-jump contribution ${ }^{10}-\sigma_{x y}^{\mathrm{VH}}$ can be smaller than $4 e^{2} / h$ and $\sigma_{x x}$. In such a case, we can keep the above-described analogy with the anomalous Hall effect. Further experimental and theoretical investigations are needed into the valley Hall effect in the insulating regime $\mathrm{e}^{30}$, where conventional formulae are not applicable.

We finally exclude another scenario that might account for the $R_{\mathrm{NL}}$ observed here. In the gap of bilayer graphene, the presence of localized states along the edge resulting from the topological property of BLG has been predicted theoretically ${ }^{31}$. This might also contribute to the nonlocal transport. With a large displacement field or large bandgap, the bulk shunting effect is small and the conduction becomes dominated by the edge transport. In such a case, $R_{\mathrm{NL}}$ should be proportional to the local resistance obtained by a four-terminal measurement (Supplementary Section X). This linear scaling does not fit any of the observed features (demonstrated in Fig. 4). We draw a linear scaling line in blue in Fig. 4, but this does not fit any of the observed features. Even when we consider the effect of bulk shunting, we find that the scaling is far from the cubic line (Supplementary Section X). So we exclude the possibility of edge transport as the origin of the observed $R_{\mathrm{NL}}$ and conclude that it comes from the bulk valley current in the gap. Also the transport through the localized states along the edge was disproved by the measurement on a Corbino geometry device ${ }^{19}$.

We used a dual-gated BLG in the Hall bar geometry to electrically control the broken inversion symmetry of BLG, and hence the valley degree of freedom. We observed a large nonlocal resistance in the insulating regime at $70 \mathrm{~K}$ and revealed a cubic scaling between the nonlocal resistance and the local conductivity as an indication of pure valley current flow. The valley current is fully controlled by electrical gating, with the bandgap, the Fermi level and broken inversion symmetry as parameters. This will allow further studies on the underlying physics of the valley current, in particular for $\sigma_{x x}<\sigma_{x y}^{\mathrm{VH}}$, as well as applications for non-dissipative 
electronic devices. While preparing the manuscript, observation of the topological valley current along an AB-BA stacking domain wall in bilayer graphene has been reported ${ }^{32}$. This topological valley current along the domain wall also originates from the non-zero valley Hall conductivity (or non-zero valley Chern number) in the gap of bilayer graphene with broken spatial inversion symmetry.

Note added in proof: We became aware that there is similar work related to valley current transport in dual-gated bilayer graphene ${ }^{33}$.

\section{Methods}

Methods and any associated references are available in the online version of the paper.

Received 27 January 2015; accepted 8 October 2015; published online 16 November 2015

\section{References}

1. Jungwirth, T., Wunderlich, J. \& Olejník, K. Spin Hall effect devices. Nature Mater. 11, 382-390 (2012).

2. Yao, W., Xiao, D. \& Niu, Q. Valley-dependent optoelectronics from inversion symmetry breaking. Phys. Rev. B 77, 235406 (2008).

3. Cao, T. et al. Valley-selective circular dichroism of monolayer molybdenum disulphide. Nature Commun. 3, 887 (2012).

4. Zeng, H., Dai, J., Yao, W., Xiao, D. \& Cui, X. Valley polarization in $\mathrm{MoS}_{2}$ monolayers by optical pumping. Nature Nanotech. 7, 490-493 (2012).

5. Mak, K. F., He, K., Shan, J. \& Heinz, T. F. Control of valley polarization in monolayer $\mathrm{MoS}_{2}$ by optical helicity. Nature Nanotech. 7, 494-498 (2012).

6. Li, Y. et al. Valley splitting and polarization by the Zeeman effect in monolayer $\mathrm{MoSe}_{2}$. Phys. Rev. Lett. 113, 266804 (2014).

7. MacNeill, D. et al. Breaking of valley degeneracy by magnetic field in monolayer $\mathrm{MoSe}_{2}$. Phys. Rev. Lett. 114, 037401 (2015).

8. Srivastava, A. et al. Valley Zeeman effect in elementary optical excitations of monolayer $\mathrm{WSe}_{2}$. Nature Phys. 11, 141-147 (2015).

9. Aivazian, G. et al. Magnetic control of valley pseudospin in monolayer $\mathrm{WSe}_{2}$. Nature Phys. 11, 148-152 (2015).

10. Xiao, D., Yao, W. \& Niu, Q. Valley-contrasting physics in graphene: Magnetic moment and topological transport. Phys. Rev. Lett. 99, 236809 (2007).

11. Mak, K. F., McGill, K. L., Park, J. \& McEuen, P. L. The valley Hall effect in MoS transistors. Science 344, 1489-1492 (2014).

12. Gorbachev, R. V. et al. Detecting topological currents in graphene superlattices. Science 346, 448-451 (2014).

13. Nagaosa, N., Sinova, J., Onoda, S., MacDonald, A. H. \& Ong, N. P. Anomalous Hall effect. Rev. Mod. Phys. 82, 1539-1592 (2010).

14. Xiao, D., Chang, M.-C. \& Niu, Q. Berry phase effects on electronic properties Rev. Mod. Phys. 82, 1959-2007 (2010).

15. Oostinga, J. B., Heersche, H. B., Liu, X., Morpurgo, A. F. \& Vandersypen, L. M. K. Gate-induced insulating state in bilayer graphene devices. Nature Mater. 7, 151-157 (2007).

16. Zhang, Y. et al. Direct observation of a widely tunable bandgap in bilayer graphene. Nature 459, 820-823 (2009).

17. Zou, K. \& Zhu, J. Transport in gapped bilayer graphene: The role of potential fluctuations. Phys. Rev. B 82, 081407 (2010).

18. Taychatanapat, T. \& Jarillo-Herrero, P. Electronic transport in dual-gated bilayer graphene at large displacement fields. Phys. Rev. Lett. 105, 166601 (2010)

19. Yan, J. \& Fuhrer, M. S. Charge transport in dual gated bilayer graphene with Corbino geometry. Nano Lett. 10, 4521-4525 (2010).
20. Koshino, M. Electronic transport in bilayer graphene. New J. Phys. 11, 095010 (2009).

21. Zhang, F., Jung, J., Fiete, G. A., Niu, Q. \& MacDonald, A. H. Spontaneous quantum Hall states in chirally stacked few-layer graphene systems. Phys. Rev. Lett. 106, 156801 (2011).

22. Valenzuela, S. O. \& Tinkham, M. Direct electronic measurement of the spin Hall effect. Nature 442, 176-179 (2006).

23. Abanin, D. A., Shytov, A. V., Levitov, L. S. \& Halperin, B. I. Nonlocal charge transport mediated by spin diffusion in the spin Hall effect regime. Phys. Rev. $B$ 79, 035304 (2009).

24. Brüne, C. et al. Evidence for the ballistic intrinsic spin Hall effect in $\mathrm{HgTe}$ nanostructures. Nature Phys. 6, 448-454 (2010).

25. Abanin, D. A. et al. Giant nonlocality near the Dirac point in graphene. Science 332, 328-330 (2011)

26. Balakrishnan, J., Koon, G. K. W., Jaiswal, M., Neto, A. H. C. \& Özyilmaz, B. Colossal enhancement of spin-orbit coupling in weakly hydrogenated graphene. Nature Phys. 9, 284-287 (2013).

27. Dean, C. R. et al. Boron nitride substrates for high-quality graphene electronics. Nature Nanotech. 5, 722-726 (2010).

28. Ohta, T., Bostwick, A., Seyller, T., Horn, K. \& Rotenberg, E. Controlling the electronic structure of bilayer graphene. Science 313, 951-954 (2006).

29. Tikhonenko, F. V., Horsell, D. W., Gorbachev, R. V. \& Savchenko, A. K. Weak localization in graphene flakes. Phys. Rev. Lett. 100, 056802 (2008).

30. Lensky, Y. D., Song, J. C. W., Samutpraphoot, P. \& Levitov, L. S. Topological valley currents in gapped Dirac materials. Phys. Rev. Lett. 114, 256601 (2015)

31. Li, J., Martin, I., Büttiker, M. \& Morpurgo, A. F. Topological origin of subgap conductance in insulating bilayer graphene. Nature Phys. 7, 38-42 (2010).

32. Ju, L. et al. Topological valley transport at bilayer graphene domain walls. Nature 520, 650-655 (2015).

33. Sui, M. et al. Gate-tunable topological valley transport in bilayer graphene. Nature Phys. http://dx.doi.org/10.1038/nphys3485 (2015).

\section{Acknowledgements}

We acknowledge fruitful discussion with L. S. Levitov, J. C. W. Song, M. Koshino M. Ezawa and N. Nagaosa. Y.S. acknowledges support from Japan Society for the Promotion of Science (JSPS) Research Fellowship for Young Scientists and JSPS Program for Leading Graduate Schools (MERIT). M.Y., K.W. and T.T. acknowledge support from JSPS Grant-in-Aid for Scientific Research on Innovative Areas 'Science of Atomic Layers'. M.Y. acknowledges support from Canon Foundation. I.V.B. acknowledges support from JSPS International Research Fellowship. K.W. and T.T. acknowledge support from the Elemental Strategy Initiative conducted by the MEXT, Japan. T.T. acknowledges support from JSPS Grant-in-Aid for Scientific Research A (No. 26248061) and JSPS Innovative Areas 'Nano Informatics' (No. 25106006). S.T. acknowledges support from DFG-JST joint research project 'Topological Electronics' and JSPS Grant-in-Aid for Scientific Research S (No. 26220710).

\section{Author contributions}

Y.S. conceived the experiment, designed the experiment with M.Y., fabricated the samples, conducted measurements and analysis, interpreted the data with M.Y., and wrote the manuscript with M.Y., I.V.B. and S.T. I.V.B. contributed to the measurements. T.T. and K.W. synthesized h-BN samples. M.Y. and S.T. directed the research. All authors discussed the results and the manuscript.

\section{Additional information}

Supplementary information is available in the online version of the paper. Reprints and permissions information is available online at www.nature.com/reprints. Correspondence and requests for materials should be addressed to M.Y. or S.T.

\section{Competing financial interests}

The authors declare no competing financial interests. 


\section{Methods}

We used a mechanical exfoliation technique to prepare bilayer graphene (BLG) and h-BN flakes. The number of layers in each graphene flake on the $\mathrm{SiO}_{2} / \mathrm{Si}$ substrate was identified by optical contrast. The $\mathrm{SiO}_{2}$ was $285 \mathrm{~nm}$ thick, the Si was heavily p-doped and used for back gating. We transferred the $\mathrm{BLG}$ flakes onto h-BN flakes prepared on $\mathrm{SiO}_{2}$ using the PMMA transfer technique reported in ref. 34 . Then Ti/ $\mathrm{Au}(10 \mathrm{~nm} / 190 \mathrm{~nm})$ was deposited to make Ohmic contacts. BLG was etched into a Hall bar by means of an Ar plasma. After each transfer and lithography step, except for the step between the Ohmic contact deposition and Ar plasma etching, the device was annealed at $300^{\circ} \mathrm{C}$ in an $\mathrm{Ar} / \mathrm{H}_{2}$ atmosphere for a few hours to remove the resist residue. However, the PMMA residue could not be completely removed by annealing, so we used a mechanical cleaning technique ${ }^{35-37}$ utilizing an AFM in tapping mode. After shaping the Hall bar, an h-BN flake was transferred to the top of the BLG/h-BN stacking layer. Finally, Ti/ $\mathrm{Au}(10 \mathrm{~nm} / 190 \mathrm{~nm})$ was deposited onto the h-BN/BLG/h-BN stacking layer to make the top gate. The thicknesses of the top and bottom h-BN layers measured by AFM were $21 \mathrm{~nm}$ and $35 \mathrm{~nm}$, respectively.
Measurements were made using a low-frequency (around $1 \mathrm{~Hz}$ ) lock-in technique. We found that current leakage through the input impedance of the voltage amplifiers causes an artefact in the nonlocal measurement. However, by using a simple circuit model, we confirmed that the error was not significant in this measurement (see Supplementary Section I). For the measurement shown in the inset of Fig. 4, we used home-made voltage amplifiers with a high input impedance to suppress the artefact further.

\section{References}

34. Taychatanapat, T., Watanabe, K., Taniguchi, T. \& Jarillo-Herrero, P. Quantum Hall effect and Landau-level crossing of Dirac fermions in trilayer graphene. Nature Phys. 7, 621-625 (2011).

35. Jalilian, R. et al. Scanning gate microscopy on graphene: Charge inhomogeneity and extrinsic doping. Nanotechnology 22, 295705 (2011).

36. Goossens, A. M. et al. Mechanical cleaning of graphene. Appl. Phys. Lett. 100, 073110 (2012)

37. Lindvall, N., Kalabukhov, A. \& Yurgens, A. Cleaning graphene using atomic force microscope. J. Appl. Phys. 111, 064904 (2012). 